\title{
Diagnostic CT: Are Patients Adequately Informed?
}

\author{
John Coyne ${ }^{1}$, Angela Brent ${ }^{2}$, Ewan O'Farrell ${ }^{1}$ \\ ${ }^{1}$ Queen Alexcandra Hospital, Portsmouth, UK \\ ${ }^{2}$ Royal Bournemouth Hospital Foundation Trust, Bournemouth, UK \\ Email: \{johnprcoyne, abrent\}@doctors.net.uk, Ewan.O’farrell@porthosp.nhs.uk
}

Received April 8, 2012; revised May 22, 2012; accepted June 13, 2012

\begin{abstract}
AIM: CT scanning is a widely utilised effective diagnostic tool. We aimed to establish whether patients are adequately informed prior to undergoing CT investigations. Methods: All adult patients with mental capacity attending the department for a CT study over a week period were invited to fill out a brief questionnaire prior to their scan. Results: 57 patients returned completed questionnaires. Overall $23 \%$ of patients were unsure or incorrect about the type of scan scheduled. Of patients attending with a new condition, $46 \%$ of them did not know their provisional diagnosis. Only 32 $\%$ of patients had insight into how a CT scan worked. This was taken to mean that the patient had included some reference to either x-rays, radiation or cross sectional imaging. Only 23\% of patients were aware of potential complications of CT scans. Conclusion: Although written consent prior to CT scan is probably a step too far in providing patients with relevant risks/benefits of their investigation, we propose all patients undergoing CT have prior access to a written information sheet should they wish to be further informed regarding their procedure.
\end{abstract}

Keywords: CT; Consent; Information

\section{Introduction}

CT scanning is a diagnostic tool used by medical pracitioners on a daily basis. It is an extremely effective and increasingly utilised method of imaging [1]. Its benefits are well established; CT is quick, accurate and can avoid unnecessary invasive surgery. One study demonstrated CT to have a sensitivity of $90 \%$ for diagnosis of abdominal pain in adults and lead to a change in management in $27 \%$ [2]. A further series showed a $24 \%$ reduction in admission rates after abdominal CT was performed in the emergency department. CT scanning however does pose its own risks, namely ionizing radiation (IR) as well as potential hazards pertaining to iodinated contrast agents.

In today's medico-legal world, patients are informed and consented regarding a wide variety of hospital practices $[3,4]$. At present, patients are not routinely consented or given detailed information about their CT scan prior to attending their appointment.

This study aimed to assess patients' understanding and knowledge regarding their upcoming investigation and the associated risks.

\section{Methods}

On entering the inpatient (IP) (patients being scanned on their current hospital admission with an acute problem) and outpatient (OP) (patients referred for non-emergency or surveillance scans) CT departments of Queen Alexandra Hospital, Portsmouth, all adult patients with mental capacity over a one week period were invited to fill out a brief questionnaire (see Figure 1). The questionnaires were handed out by the radiographers in the respective waiting areas during the normal working day. The questionnaires were collected in and the results seen below. No conscious adult patients were excluded from the trial. Children were not involved in the study.

\section{Results}

There were 213 scans carried out during this period that met the inclusion criteria, 107 outpatient and 96 inpatient scans. Therefore there was a response rate of $27 \%$ in this study. The reasons for lack of completion were not recorded (Table 1).

Table 1. Questionnaire response.

\begin{tabular}{llcc}
\hline & & & \\
& $\begin{array}{c}\text { Inpatients } \\
\text { (IP) }\end{array}$ & $\begin{array}{c}\text { Outpatients } \\
\text { (OP) }\end{array}$ & Total \\
& & & \\
\hline $\begin{array}{l}\text { Number of questionnaires } \\
\text { completed }\end{array}$ & 19 & 38 & 57 \\
\end{tabular}




\section{Discussion}

The diagnostic effectiveness of CT with consequent implications on patient management has driven a dramatic increase in the numbers of CT scans being performed. It is also undoubted that a combination of other factors including; litigation fear, miscommunication and duplication of requests and non-specialist clinical review leads to over-imaging [1]. This leads to the question of radiation protection and informed decision making by patients.

Relative risk is frequently given as a justification for and against providing information to patients regarding IR exposure. The risk of 0.5 per 1000 individuals for a 10 mSv CT compares favourably with that of 1.6:1000 of a fatal pedestrian road traffic accident. Equally, against the back drop of a greater than 1:3 life time risk for development of cancer the purported 1:2000 increase risk associated with a $10 \mathrm{mSv}$ exposure becomes both less significant and difficult to prove causally [2].

Consent is required for risks pertaining to investigations or procedures that a "reasonable person would want to know" to make an informed decision. This is challenging with respect to IR as the risks are difficult to truly quantify [2-6]. There is concern that patients may withdraw their consent to a potentially lifesaving invest-

Patient Centred Practice in Diagnostic Imaging

We are currently looking into aspects of the service that we provide here in the radiology department. Our goal is to deliver high quality care, and this study aims to check that we are achieving this aim and that we are continuing to improve.

Your participation is entirely voluntary and does not affect any scan or treatment you may be having. We would be very grateful if you could answer the questions below and provide any comments you wish at the end.

1. Are you aware of what scan you have come for today? Yes/No/Not sure

2. Is this scan to follow up a condition that you are aware of already or is it a new problem? Existing condition [ ]
New problem
[ ]

3. Have your doctors told you what they are looking for with this scan? (What problem or condition they suspect might have?) Yes/No

4. Have you had any other scans recently? Yes/No

5. Have any other possible scans or tests been discussed with you? Yes/No

6. How do you think this scan works?

7. Are you aware of any risks or possible complications of this scan? Yes/No

If yes, please write below:

8. Regarding the tests and scans you have had, how would you rate the level of information you received? $(1-5,1=$ far too little, $5=$ far too much)

9. Is there anything else you would like to know about the scan you have come for?

Yes/No

If Yes, please describe:

10. Any other comments:

Figure 1. Questionnaire patient awareness of CT. tigation if informed of the very small risk of radiationinduced cancers. Counter to this argument is that patients have the right to arrive at a weighed decision, and this is no different to disclosure of risks for anaesthesia or surgery. Various studies have shown that in the most sensitive population, paediatrics, explaining the risks of CT does not dissuade parents from consenting to the investigation $[7,8]$.

The American Medical Association stipulates the key components of informed consent include a discussion regarding the patient's diagnosis, nature and purpose of the proposed treatment or procedure including risks and benefits and alternatives with concurrent risks and benefits [4].

The audit clearly demonstrated that a significant number of patients have inadequate information about the reason for and nature of the investigation for their condition. Overall $21 \%$ of patients were unsure or incorrect about the type of scan scheduled (Table 2). Of patients attending with a new condition, $46 \%$ of them did not know their provisional diagnosis. Perhaps not surprisingly, of the $64 \%$ of IP with a new acute presentation $50 \%$ of them were not aware of their provisional diagnosis. However, more unanticipated was that amongst the $31 \%$ of OP with a new condition $42 \%$ were unaware of their provisional diagnosis (Table 3 ).

There was a clear differentiation between patient's prior knowledge and appreciation of their condition and imaging between the IP and OP settings. Predictably, a high proportion (67\%) of OP scans was following up a known condition as opposed to $37 \%$ of IP scans. This in part translates to the excess of patients who were unsure or incorrect about the type of scan they were attending for in the IP population of $52 \%$ compared to $8 \%$ of OP (Tables 2, 3). Additionally, patients attending for outpatient CT receive a generic letter inviting them for an appointment. This includes the wording "CT scan” and advises them that they may receive intravenous contrast. It does not detail risks and benefits of CT imaging.

As part of the consent process available alternatives should be offered. There were $67 \%$ of patients whom had other examinations and 32\% of patients had had other investigations discussed. In the absence of full documentation about discussions with the patient this may serve as a surrogate marker of alternative and/or supplementary investigations having been performed and considered.

Where arguably the Clinical radiologist and radiology department have more of a role to play is in educating patients and clinicians about the nature of investigations and informing, where appropriate, about potential risks involved. Only $32 \%$ of patients (26\% IP/34\% OP) had insight into how a CT scan worked. This was taken to mean that the patient had included some reference to either $\mathrm{x}$-rays, radiation or cross sectional imaging. Only 
$23 \%$ of patients were aware of potential complications of CT scans (Table 4).

There was a poor uptake of questionnaires in both IP and OP settings. This is multifactorial. Many patents did not have their spectacles and were unable to read the questionnaire. In any survey, there is recognised attrition of people who do not wish to fill out supplementary information. Patients in the IP group were more acutely unwell accounting for some of the lower uptake seen in this population. Workflow in the IP setting is busier and more unpredictable lending to radiographers possibly not always handing out questionnaires.

Our results clearly demonstrate that patients undergo-

Table 2. Patient awareness of investigation type.

\begin{tabular}{lcc}
\hline & $\begin{array}{c}\text { Inpatients } \\
\text { (IP) }\end{array}$ & $\begin{array}{c}\text { Outpatients } \\
\text { (OP) }\end{array}$ \\
\hline $\begin{array}{l}\text { Patient aware of type of scan } \\
\text { attending for }\end{array}$ & $10 / 19$ & $35 / 38$ \\
$\begin{array}{l}\text { Patient unsure of type of scan } \\
\text { attending for }\end{array}$ & $5 / 19$ & $0 / 38$ \\
$\begin{array}{l}\text { Patient unaware/incorrect } \\
\text { about type of scan attending } \\
\text { for }\end{array}$ & $4 / 19$ & $3 / 38$ \\
\hline
\end{tabular}

Table 3. Information basis of patients attending for CT scan.

\begin{tabular}{lcc}
\hline & $\begin{array}{c}\text { Inpatients } \\
\text { (IP) }\end{array}$ & $\begin{array}{c}\text { Outpatients } \\
\text { (OP) }\end{array}$ \\
\hline $\begin{array}{l}\text { Investigation of pre-existing } \\
\text { condition }\end{array}$ & $7 / 19$ & $26 / 38$ \\
$\begin{array}{l}\text { New condition with patient } \\
\text { aware of provisional diagnosis }\end{array}$ & $6 / 19$ & $7 / 38$ \\
$\begin{array}{l}\text { New condition with patient } \\
\text { unaware of provisional } \\
\text { diagnosis }\end{array}$ & $6 / 19$ & $5 / 38$ \\
\hline
\end{tabular}

Table 4. Satisfying criteria for informed consent and justification of examinations.

\begin{tabular}{lcc}
\hline & $\begin{array}{c}\text { Inpatients } \\
\text { (IP) }\end{array}$ & $\begin{array}{c}\text { Outpatients } \\
\text { (OP) }\end{array}$ \\
\hline $\begin{array}{l}\text { Patient has insight into how a } \\
\text { CT scan works }\end{array}$ & $5 / 19$ & $13 / 38$ \\
$\begin{array}{l}\text { Patient aware of potential } \\
\text { complications of the scan }\end{array}$ & $4 / 19$ & $9 / 38$ \\
$\begin{array}{l}\text { Patient aware of how the result } \\
\text { of the scan will be followed up }\end{array}$ & $6 / 19$ & $25 / 38$ \\
$\begin{array}{l}\text { Patient felt they would like } \\
\text { more information }\end{array}$ & $5 / 19$ & $2 / 38$ \\
\hline
\end{tabular}

ing CT scan in our department are currently poorly informed regarding their upcoming procedure. Patients are currently not aware of the risk of ionising radiation that CT involves and its risk/benefit profile. Although patient information sheets have been written [9] they are not available in our department. If written information were to be given to patients, those who choose to do so can become more fully informed regarding their investigation.

In addition to written information, medical practitioners need to ensure that patients attending scan do so with as much information regarding potential diagnoses as the patient wishes to receive at the time of scan request. We appreciate that giving such information is not always appropriate and differentials can be extensive but at the very least, the type of scan being carried out should be known by patients prior to their imaging.

\section{Conclusions}

Although written consent prior to CT scan is probably a step too far in providing patients with relevant risks/ benefits of their investigation, we propose all patients undergoing CT have prior access to a written information sheet should they wish to be further informed regarding their procedure.

\subsection{Limitations/Improvements and Future Developments}

This study had several limitations. Although the results demonstrated clear trends, the patient groups were small and more data would have added to the power. The questionnaire included some questions that could be considered ambiguous. This was due to the necessity to keep prose to a minimum as it is well recognised that patients as a group both do not wish to read, nor comprehend extended additional materials. It was requisite to keep terms generalised in order to not skew results with leading questions. Establishing if a patient has been informed about alternative methods of imaging presented a conundrum. In the absence of a detailed discussion with each patient, it was not possible to directly obtain this information and consideration of other investigations was taken as an appreciably crude and flawed marker.

Collection of more data and redesign of the questionnaire to include more objective measurements would improve the accuracy and aid interpretation of the data.

Our institute aims to produce information pamphlets for patients to be available on the ward and in OP clinics in addition to posters in the department to facilitate informing patients. An intranet link on the hospital website is also planned to utilise multimodality approaches to gain a larger target audience.

It is also important to educate clinicians making refer- 
rals, about their duty to adequately inform patients under their care. This is allied with edicts from several professional bodies including the General Medical Council and care quality commision (CQC) concerning patient centred care. These principals stipulate that people who use NHS services are involved in and receive care, treatment and support that recognises their right to make decisions based on an explanation and discussion of theiroptions. The CQC champions patients' rights to take informed risks, while balancing the need for preference and choice with safety and effectiveness [10]. Our aim is to present the key findings from this study to clinicians at a Grand Round to highlight and disseminate these messages.

\subsection{Study Design}

The study was devised by the principal authors, Dr J. Coyne and Miss A. Brent. The questionnaire was designed by Drs Coyne and O'Farrell. Data was tabulated and analysed using Microsoft "Excel”.

\section{REFERENCES}

[1] M. Baerlocher and A. Detsky, "Discussing Radiation Risks Associated with CT Scans with Patients,” Journal of the American Medical Association, Vol. 304, No. 19, 2010, pp. 2170-2171. doi:10.1001/jama.2010.1591

[2] S. Ahn, W. Mayo-Smith, et al., "Acute Nontraumatic
Abdominal Pain in Adult Patients: Abdominal Radiography Compared with CT Evaluation,” Radiology, Vol. 225. No. 1, 2002, pp. 159-164. doi:10.1148/radiol.2251011282

[3] J. Brink, M. Goske and J. Patti, "Informed Decision Making Trumps Informed Consent for Medical Imaging with Ionizing Radiation,” Radiology, Vol. 262, No. 1, 2012, pp. 11-13. doi:10.1148/radiol.11111421

[4] R. Semelka, D. Armao, et al., "The Information Imperative: Is It Time for an Informed Consent Process Explaining the Risks of Medical Radiation?” Radiology, Vol. 262, No. 1, 2012, pp. 15-18. doi:10.1148/radiol.11110616

[5] “Good Practice Guide for Clinical Radiologists,” Royal College of Radiologists, London, 2012.

[6] W. Hendee, "Real and Perceived Risks of Medical Radiation Exposure,” Western Journal of Medicine, Vol. 138, 1983, pp. 380-386.

[7] M. Baerlocher and A. Detsky, "Informed Consent for Radiologic Procedures,” Journal of the American Medical Association, Vol. 305, No. 9, 2011, pp. 888-890.

[8] D. Larson, S. Rader, H. Forman and L. Fenton, “Original Research: Informing Parents about CT Radiation Exposure in Children: It's OK to Tell Them," American Journal of Roentgenology, Vol. 189, No. 2, 2007, pp. 271275.

[9] Clinical Radiology Patients' Liaison Group (CRPLG) of The Royal College of Radiologists. London: The RCR, December 2010.

[10] Care Quality Commission, “Guidance about Compliance Essential Standards of Quality and Safety,” 2010. 